\title{
Transmédia et valorisation du patrimoine : l'exemple de Montaigne Superstar
}

Jessica de Bideran, Mélanie Bourdaa et Justine Dujardin

\section{OpenEdition \\ Journals}

Édition électronique

URL : http://journals.openedition.org/ocim/1864

DOI : $10.4000 /$ ocim. 1864

ISSN : 2108-646X

Éditeur

OCIM

Édition imprimée

Date de publication : 1 novembre 2017

Pagination : 5-10

ISSN : 0994-1908

Référence électronique

Jessica de Bideran, Mélanie Bourdaa et Justine Dujardin, « Transmédia et valorisation du patrimoine l'exemple de Montaigne Superstar ", La Lettre de I'OCIM [En ligne], 174 | 2017, mis en ligne le 01 novembre 2018, consulté le 25 juillet 2019. URL : http://journals.openedition.org/ocim/1864 ; DOI : 10.4000/ocim. 1864

Ce document a été généré automatiquement le 25 juillet 2019

Tous droits réservés 


\title{
Transmédia et valorisation $\mathrm{du}$ patrimoine : l'exemple de Montaigne Superstar
}

\author{
Jessica de Bideran, Mélanie Bourdaa et Justine Dujardin
}

Affiche de l'évènement Montaigne Superstar, création de la bibliothèque municipale de la ville de Bordeaux à partir d'un dessin de Jul, 2016.

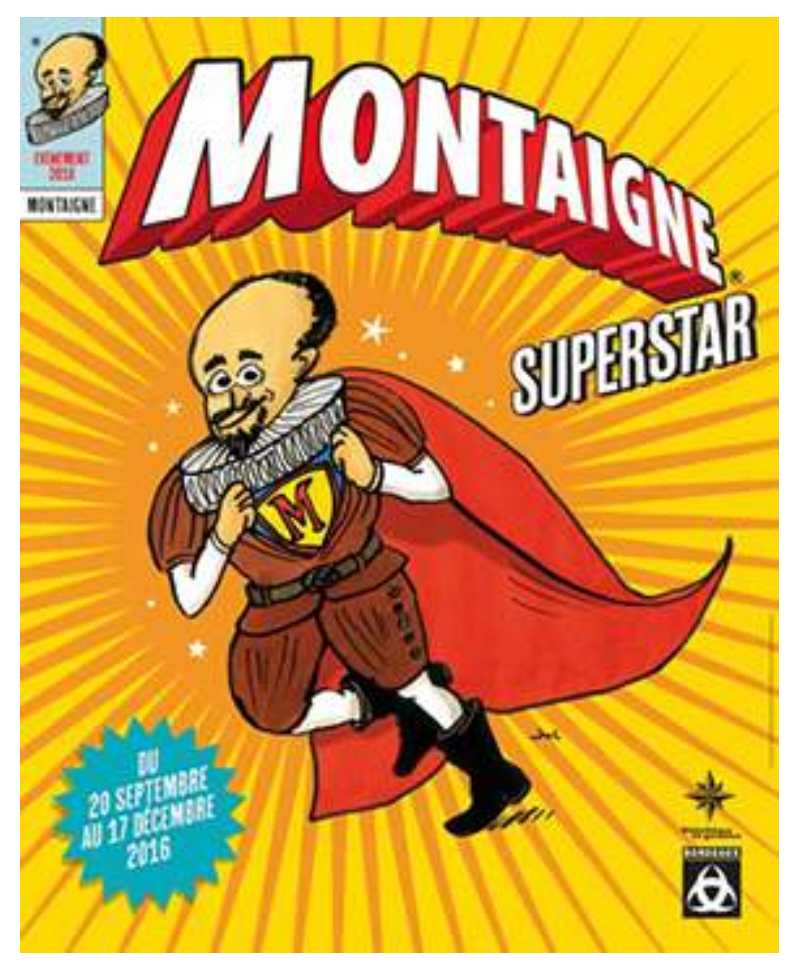

1 Faire vivre une expérience, telle semble aujourd'hui la mission assignée à la médiation culturelle. Pour cela, institutions et acteurs culturels expérimentent de nouvelles formes de médiation en proposant des expériences in situ (visites guidées et théâtralisées, 
applications de visite, escape games...) ou des expérimentations en ligne (réseaux sociaux, blogs, webdocumentaires...). Mais loin de s'opposer, ces diverses pratiques ont aujourd'hui tendance à se rejoindre et à se recouper grâce, par exemple, aux activités d'animation mises en place par les community managers de plus en plus présents dans les institutions culturelles (Magro, 2015).

Ces nouvelles stratégies de valorisation s'inscrivent dans une convergence culturelle et technologique parfaitement décrite par Henry Jenkins en 2006 (Jenkins, 2006). À partir de l'analyse des pratiques de dissémination de contenus culturels proposées par l'industrie $\mathrm{du}$ divertissement hollywoodienne, celui-ci propose en effet de définir le concept de transmedia storytelling (narration transmédia) pour nommer ces nouvelles pratiques de communication. Pour ces acteurs il s'agit de promouvoir leurs "marques" en créant un univers et en diffusant des récits dont les divers éléments narratifs sont dispersés sur différents supports, numériques et analogiques, pour créer une expérience unifiée et coordonnée. Mais si ces techniques s'adaptent parfaitement aux logiques des industries culturelles, qui disposent de moyens financiers considérables comme d'importantes communautés de fans susceptibles de servir de caisses de résonance, celles-ci peuventelles s'appliquer à l'univers du patrimoine et de ses acteurs ? La réalité du terrain de ce secteur, avec les contraintes qui lui sont propres, est-elle compatible avec ces procédures ? Et quelles peuvent en être les impacts sur la médiation culturelle et l'appropriation, par les publics, des contenus et savoirs véhiculés ? L'étude d'un projet particulier élaboré par le service des Fonds anciens et patrimoniaux de la bibliothèque municipale de Bordeaux, permet de répondre à ces questions et d'évaluer ainsi la pertinence de telles pratiques pour la médiation culturelle.

\section{Narration transmédia et patrimoine, ou comment engager les publics?}

3 En suivant les principes posés par Jenkins, les stratégies transmédiatiques visent donc à créer des univers narratifs cohérents en dispersant les éléments de l'histoire racontée sur différentes plateformes médiatiques ou non médiatiques, le tout dans le but d'engager les publics dans une co-construction et une co-participation. C'est cet "art du worldbuilding" (Long, 2007) qui constitue la pierre angulaire de ces dispositifs. Permettant de construire un univers complexe, riche et foisonnant en contenus, le principe d'une narration transmédia paraît ainsi bien adapté à la valorisation patrimoniale où l'on retrouve chez certains publics cette "passion de l'encyclopédiste avide de connaissance sur un monde... ou la passion du collectionneur qui acquiert plus d'objets liés à ce monde" (Ryan, 2017).

4 À l'échelle internationale, il est possible de recenser une trentaine de projets à vocation patrimoniale exploitant des éléments narratifs appartenant à un même univers mais éclatés sur différents supports (site internet, réseaux sociaux, application de visite, expositions...) $)^{1}$. Pour autant, tous ces projets n'invitent pas à une même participation des publics ni même ne développent de véritables interactions entre "experts" et "profanes". Il est ainsi intéressant de constater que les expérimentations principalement centrées sur une figure historique, telles Le Roi est Mort ${ }^{2}$ développé par le château de Versailles ou encore Le Dernier Gaulois ${ }^{3}$ diffusé sur France Télévision, s'appuient de préférence sur une stratégie de la production vers les publics selon un schéma communicationnel descendant classique. Tout se passe ici comme si la figure historique présentée par ces dispositifs 
revêtait un caractère sacré, une sorte d'aura, qu'il ne convenait pas de questionner et sur laquelle seuls les discours des professionnels auraient voix au chapitre.

La scénographie de l'exposition Montaigne Superstar

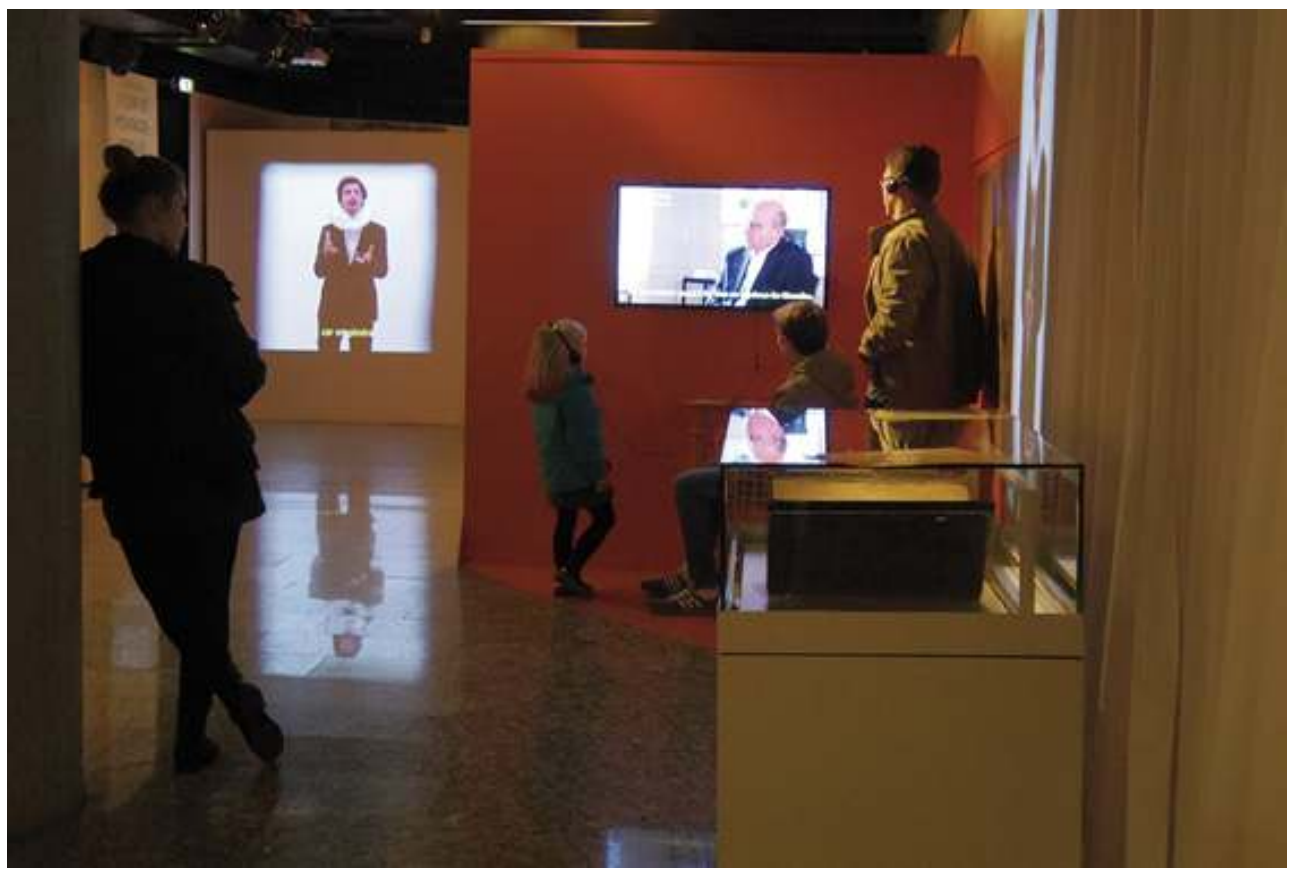

(c) Jessica de Bideran

5 À l'inverse, les projets axés sur la valorisation du patrimoine monumental et ceux centrés sur le patrimoine immatériel semblent plus enclins à favoriser la participation des publics, notamment par la co-construction de la narration.

6 Le Défi des bâtisseurs ${ }^{4}$ est à cet égard éclairant ; il s'agit là d'un dispositif produit principalement par Arte en 2013, en association avec différents studios et structures institutionnelles, et visant à valoriser la cathédrale de Strasbourg. L'ensemble des médias développés, un docu-fiction en relief, une application mobile de visite et un web documentaire interactif, sont en effet intimement liés par une segmentation de la narration et par un enchevêtrement des actions proposées aux publics. Ces derniers sont par exemple incités à construire la tour manquante de la cathédrale grâce à l'outil interactif développé pour le web documentaire, le "tower builder". Car c'est bien l'internaute qui est ici mis au centre du dispositif, et cela dès son entrée dans l'univers narratif ; par le biais d'une vision en caméra subjective, il/elle est ainsi recruté(e) comme membre d'un cabinet d'architectes afin de dessiner les plans et les élévations de cette seconde tour. S'en suit une enquête ludique qui invite l'usager à chercher des indices historiques au sein des ressources documentaires mises en ligne, à interviewer des personnages clés de l'histoire de ce monument animés dans le cadre du docu-fiction et, enfin, à se rendre sur place pour débloquer, grâce à l'application de visite, des éléments narratifs supplémentaires. 


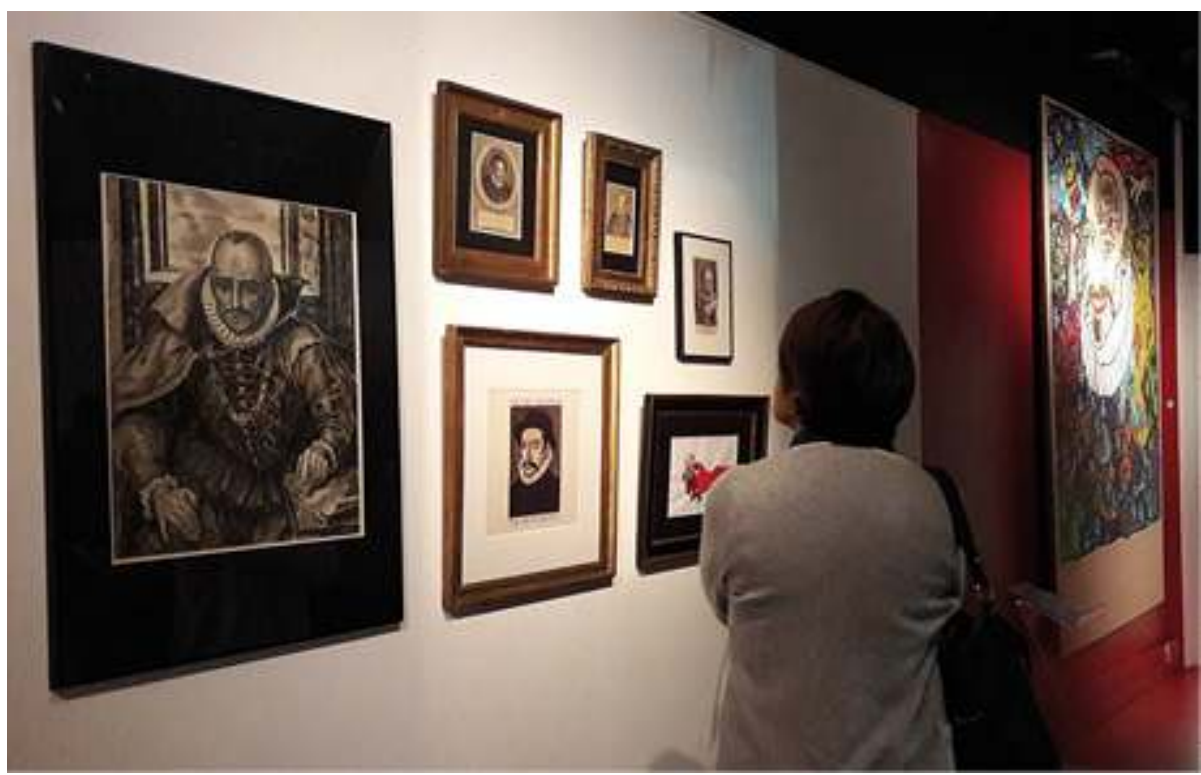

(c) Jessica de Bideran

Cette expérimentation constitue ainsi une sorte de parangon de la narration transmédia appliquée à la médiation patrimoniale. Les producteurs ont en effet su créer un univers narratif complet permettant d'immerger les publics dans l'histoire racontée tout en délivrant des informations complexes sur le monument et sa construction. Des dispositifs médiatiques plus classiques, tels que le docu-fiction, ont d'autre part été complétés par des dispositifs plus originaux, à l'image du "tower builder" mais aussi des réseaux sociaux et de l'application mobile, incitant les publics à construire une partie de l'histoire et à collaborer. Se manifeste ici une forme de culture participative, "une culture favorable à l'expression artistique et l'engagement civique, un soutien important à la création et au partage des créations, et une sorte de mentorat informel où ce qui est connu par les plus initiés est transmis aux novices. Une culture de la participation est aussi une culture dans laquelle les membres croient à l'importance de leurs contributions, et ressentent des liens sociaux avec les autres membres (au moins ils se soucient de ce que les autres pensent de leurs créations)" (Jenkins, Ito, Boyd, 2015 : 4). Exploitant la réputation, artistique, historique et touristique de la cathédrale de Strasbourg, ce projet a su, à ses débuts, impulser un véritable élan de participation chez les internautes... élan qui certes s'épuise avec le temps mais dont les créations continuent cependant à exister en ligne tout en s'enrichissant de nouvelles expériences développées pour la visite du monument.

D’autres expériences peuvent être relevées dans le domaine du patrimoine immatériel et valorisant des traditions, des langues ou encore des légendes de pays. Objets culturels non tangibles, flottant dans le temps et dans l'espace, ces derniers perdent en effet parfois leur force d'une génération à l'autre, surtout lorsqu'ils dépendent essentiellement de la tradition orale. Un dispositif médiatique les aide à se concrétiser et agit alors comme une archive, en conservant la trace de ces coutumes. La disparition et le manque d'intérêt parfois manifesté par les plus jeunes pour ce patrimoine immatériel peuvent en outre être contrebalancés par une démarche créative mise en œuvre dans le cadre d'un projet transmédia. 
$9 \quad$ La Chasse aux Légendes ${ }^{5}$ créée par un studio de production bordelais, s'attaque pour sa part à la culture basque et a été développé dans cet esprit. Accessible depuis une application mobile, une narration se déploie pour chaque légende à travers une bande dessinée interactive avant d'inciter les jeunes joueurs et leurs familles à se rendre sur des sites naturels du pays basque pour chasser l'une des créatures fantastiques évoquées afin, tout comme dans le célèbre jeu Pokemon Go, de la capturer. Mais La Chasse aux Légendes va plus loin puisque, outre le fait que toutes ces figures fantastiques soient parfaitement référencées dans la culture basque, une seconde narration encadre celle des légendes, écrite autour d'un personnage central, un esprit de la nature, que l'on retrouve dans un conte audio ainsi que dans un livre. Si les publics touristes sont actifs par leur déplacement et la capture des légendes, des publics de scolaires ont également été impliqués dans l'écriture de nouvelles légendes, à partir là encore d'antiques récits basques et de déplacements dans des lieux géographiques. Intégrés à la stratégie globale, ces éléments narratifs ont été réalisés par des lycéens bayonnais dans le cadre de projets d'Éducation artistique et culturelle. Ils traduisent parallèlement la dimension vivante de cette culture traditionnelle qui réussit à réunir patrimonialisation, création et appropriation...

\section{Montaigne Superstar ou comment faire du transmédia sans le savoir?}

10 Associer patrimoine, création et appropriation, tel était aussi l'objectif de la récente manifestation patrimoniale de la bibliothèque municipale de Bordeaux qui organise tous les deux ans un évènement d'ampleur destiné à valoriser le riche patrimoine conservé en réserves, au premier rang duquel se trouvent les fonds des fameux "3M" : Mauriac, Montesquieu et Montaigne.

11 Concrètement, le premier objectif de Montaigne Superstar était d'élaborer une manifestation qui reflète les métiers, activités et approches patrimoniales d'aujourd'hui. Montaigne constituait de ce fait une figure parfaite : parce que ses œuvres font partie des trésors les plus précieux conservés à Bordeaux, celles-ci ont fait l'objet de plusieurs expositions au cours du $\mathrm{XX}^{\mathrm{e}}$ siècle et l'idée, pour ce projet, était de faire une exposition "datée 2016" qui parle à nos contemporains afin de leur faire prendre conscience que le patrimoine écrit s'adresse à tous, qu'il est accessible et qu'il peut trouver une résonance aujourd'hui. Le deuxième objectif était de rendre cohérentes les collections proposées au public, de créer des correspondances entre hier et aujourd'hui, par les supports et services proposés dans tout le réseau des bibliothèques, mais aussi par le biais d'activités créatives, participative et festives. Si de nombreux services de la bibliothèque participaient à la préparation de la programmation, des activités devaient également être organisées dans plusieurs établissements et la ville devenir le théâtre de diverses manifestations (rallyes, visites guidées...). Enfin, le troisième objectif était de redonner à voir et à comprendre, à tous et pour tous, ce patrimoine écrit conservé en réserve la plupart du temps et donc largement ignoré... Pour cela, il convenait de dépasser la simple "monstration", voire la "démonstration", qui maintient généralement les publics dans une attitude passive de contemplation... Si les usagers qui fréquentent une bibliothèque de lecture publique s'approprient le lieu, ses collections et ses services, la même chose devait être faite avec le patrimoine écrit afin que ces derniers se sentent véritablement impliqués, immergés, actifs, accompagnés. 
L'espace permettant de jouer au jeu de plateau créé par l'équipe de bibliothécaires dans le cadre de l'exposition Montaigne Superstar.

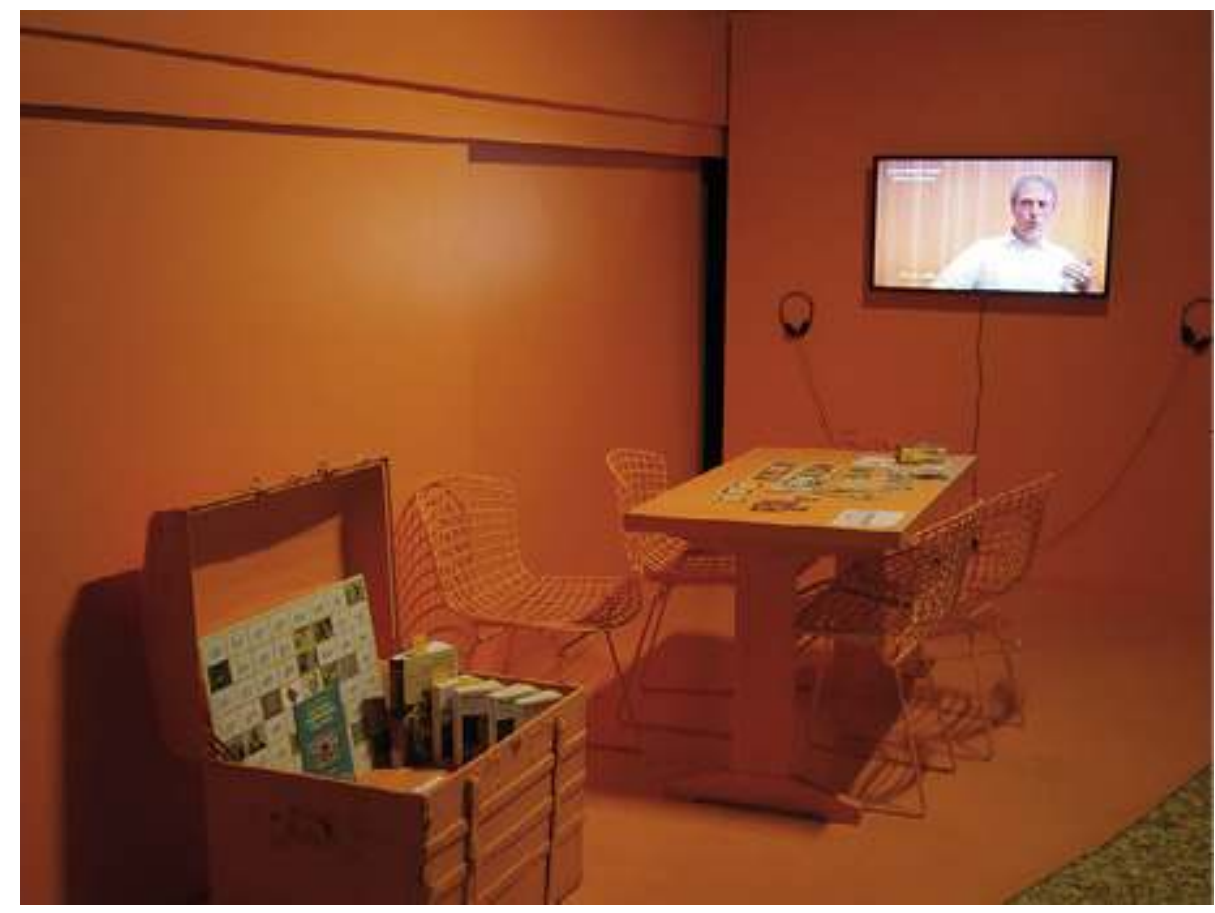

(C) Jessica de Bideran

12 Mais exposer du patrimoine écrit, c'est exposer des textes que par ailleurs on manipule, on lit, on emporte avec soi... La barrière de la vitrine, la frustration de ne voir qu'une seule page de tout un livre doit donc être dépassée ; cela a pu être le cas ici grâce notamment au partenariat mis en place avec la Bibliothèque nationale de France pour numériser "l'Exemplaire de Bordeaux", exemplaire de 1588 entièrement annoté de la main de Montaigne, mais aussi par l'enrichissement des contenus sur internet.

Car la principale difficulté de cette manifestation était la matière première : une œuvre monumentale qui en impressionne plus d'un. Le sujet n'était pas non plus très à la mode, malgré le capital sympathie de la figure de Montaigne. Un rapide sondage au sein de l'équipe projet a ainsi révélé que si tout le monde connaît Montaigne, peu le lisent et les souvenirs associés au philosophe sont souvent liés à de douloureuses expériences scolaires... Or les idées, les thèmes et ses textes même, malgré cet abord peu commode, sont vigoureux, vivants et parlent à notre époque. Comment dès lors intéresser un large public à un matériau à première vue un peu austère ? En jouant peut-être de ce décalage... Et comment donner envie aux usagers de la bibliothèque de lire Montaigne, de transformer un événement public en expérience intime ? En racontant sans doute une histoire qui devienne celle du public...

14 C'est ainsi que s'est progressivement imposée cette association de mots qui à elle seule résume l'esprit de la manifestation : Montaigne Superstar, ou comment un noble périgourdin est devenu une figure littéraire mondiale, un homme de son temps profondément original, si proche et si lointain à la fois. Et si le contenu de la manifestation est rigoureusement scientifique, le ton lui se veut léger, actuel, avec une touche d'humour et d'autodérision sur nos propres canaux de médiatisation, de 
communication et notre façon bien actuelle de re-fabriquer la culture, à l'image des mécanismes d'une narration transmédia.

Ouverte aux publics entre septembre et décembre 2016, l'exposition Montaigne Superstar s'est ainsi insérée au sein d'une manifestation bien plus large conçue dans une visée de dissémination de contenus narratifs caractéristique d'une stratégie transmédiatique. Celle-ci s'appuie en effet sur un emboitement des contenus et systèmes de médiation produits et diffusés selon des temporalités distinctes. Audiovisuels, table tactile de consultation de corpus documentaires, jeu de plateau exploitable dans le cadre de l'exposition ou au sein des bibliothèques de quartier partenaires de l'événement, ont été devancés par toute une série de déclinaisons numériques des contenus (profil Facebook, blog, chaîne Youtube...) toujours consultables aujourd'hui en ligne.

L'impression qui ressort à la lecture des nombreux accès aux contenus historiques et patrimoniaux proposés est celle d'une offre extrêmement riche, pléthorique et par conséquent complexe à appréhender dans son ensemble. Mais le public pouvait faire son choix dans les propositions en ne s'engageant pas forcément sur tous les tableaux, ce qui a permis de proposer plusieurs niveaux d'entrée dans la manifestation selon les intérêts des publics et leur appétence pour les supports et médias utilisés. Certains publics ont ainsi été plus à l'aise avec les cartels écrits qu'avec les audiovisuels disponibles dans l'exposition, d'autres se contentant de suivre la page Facebook quand d'autres ont été plus attirés par les activités ludiques (jeu de plateau et murder party)... Par ces multiples possibilités, certes pas toujours évidentes à combiner, la manifestation a toutefois réussi à réunir plusieurs types de publics. Des familles habituées à fréquenter la bibliothèque, de jeunes adultes attirés par les jeux et les visiteurs plus traditionnels des expositions artistiques et patrimoniales ont ainsi majoritairement manifesté leur plaisir et leur satisfaction. Car la manifestation était conçue avant tout pour laisser au public le choix de son usage et de son niveau d'implication tout en retirant de chaque participation des informations historiques. Et l'exposition syncrétise finalement ces expériences et les usages qui se sont progressivement mis en place en ligne et in situ : les agents assurant l'accueil dans les salles ont ainsi remarqué des visiteurs revenant à plusieurs reprises pour approfondir tel aspect, s'installer pour feuilleter les livres présentés, ou voir les vidéos... 
L'atelier pédagogique consacré à la découverte de l'histoire de l'imprimerie organisé dans le cadre de l'exposition Montaigne Superstar.

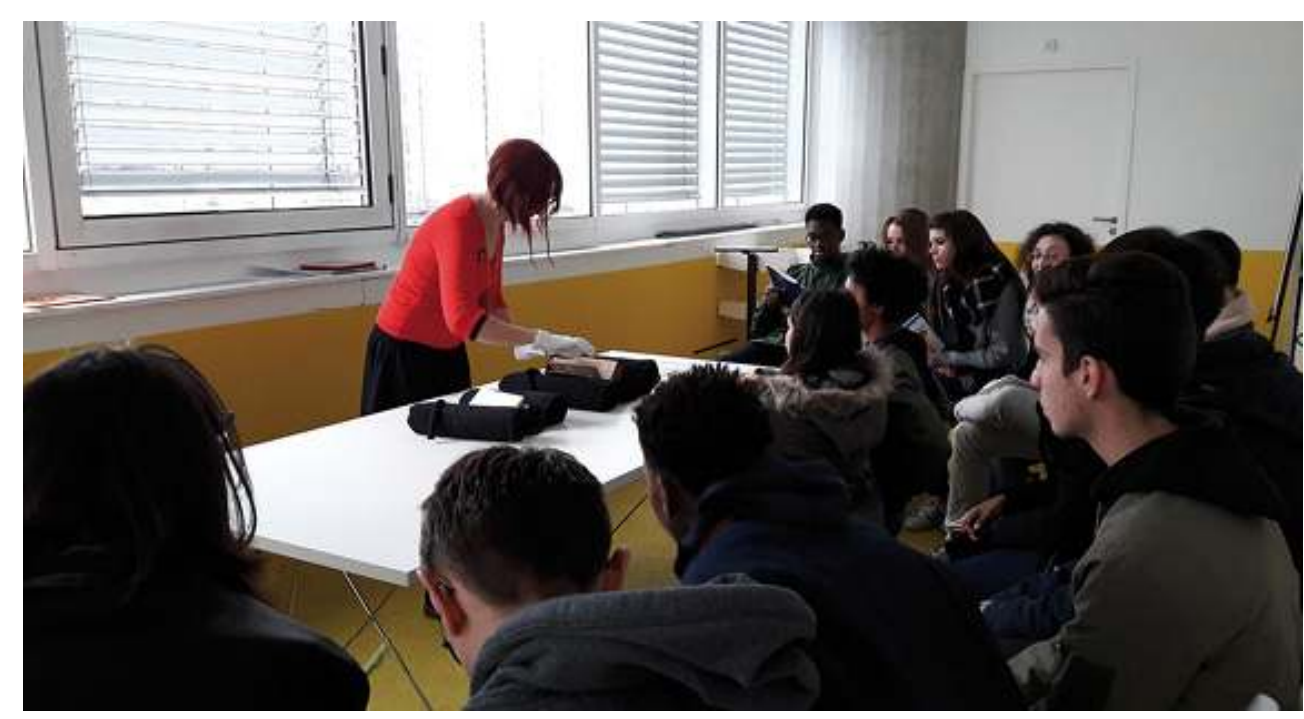

(C) Jessica de Bideran

\section{Jeunes publics et transmédia, ou comment repenser la médiation pédagogique?}

17 Considérée donc comme un "succès", du fait de ses importantes retombées médiatiques, de la hausse exceptionnelle du nombre de visiteurs et du fort intérêt manifesté par les visiteurs, Montaigne Superstar apparaît comme une manifestation ayant réussi à fédérer des publics par une politique de communication efficace. Mais l'analyse de cet événement à l'aune du concept de transmedia storytelling permet d'aller plus loin en appréhendant notamment l'impact d'un tel processus de diffusion sur les publics et, finalement, sur l'objet patrimonial. Pour proposer un début de réponse à cette problématique, nous avons donc mené, entre octobre et décembre 2016, une étude dont l'angle d'analyse était les publics scolaires ${ }^{6}$.

18 Une narration transmédia se caractérisant en effet par la construction d'un univers narratif dans lequel les publics sont poussés à s'engager grâce, notamment, à la fragmentation des contenus sur différents support, les objectifs de l'étude de terrain consistaient d'une part à qualifier la réception du monde construit par l'équipe auprès des publics jeunes et, d'autre part, à évaluer l'intégration des différents discours développés (en ligne et in situ) au sein du dispositif de médiation que représentent les ateliers pédagogiques. En interrogeant les enseignants nous avons également cherché à observer et évaluer l'intégration du concept de narration transmédia dans leur démarche pédagogique, en jugeant par exemple de leur exploitation des dispositifs en ligne pour faciliter l'appropriation et la création de nouveaux contenus par leurs élèves.

Lors de chaque séquence observée à la bibliothèque, la découverte pédagogique de Montaigne Superstar s'est toujours articulée en deux volets : isolés dans une pièce dédiée, les élèves étaient d'abord confrontés à des ouvrages du XVI ${ }^{e}$ siècle afin d'appréhender le concept de patrimoine écrit. Après ce premier temps d'échanges avec les bibliothécaires, les élèves étaient ensuite invités à suivre le parcours scénographique de l'exposition tout 
en répondant à un quizz proposé au début de la visite et élaboré par les équipes de la bibliothèque. C'est après avoir observé chacun de ces temps de médiation que nous avons pu interroger les lycéens et les enseignants afin de mieux comprendre les raisons de leur adhésion au projet ou, au contraire, les aspects mal perçus.

Extrait de Les Transformations de Montaigne, œuvre collective réalisée par des jeunes en insertion professionnelle au centre EPIDE (Établissement pour l'insertion par l'emploi) de Bordeaux, inspirés par Les Essais de Montaigne.

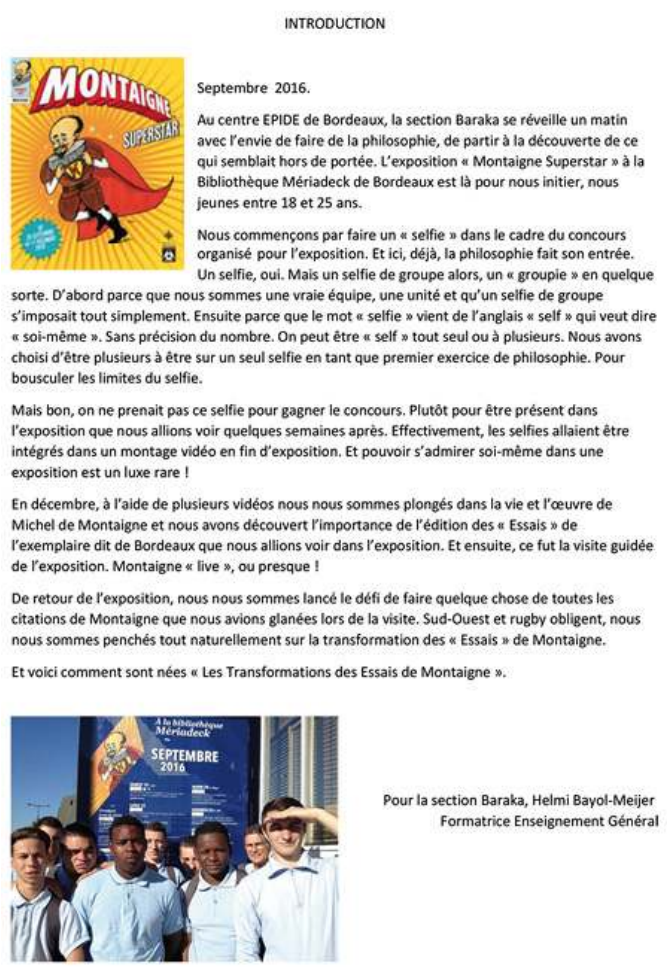




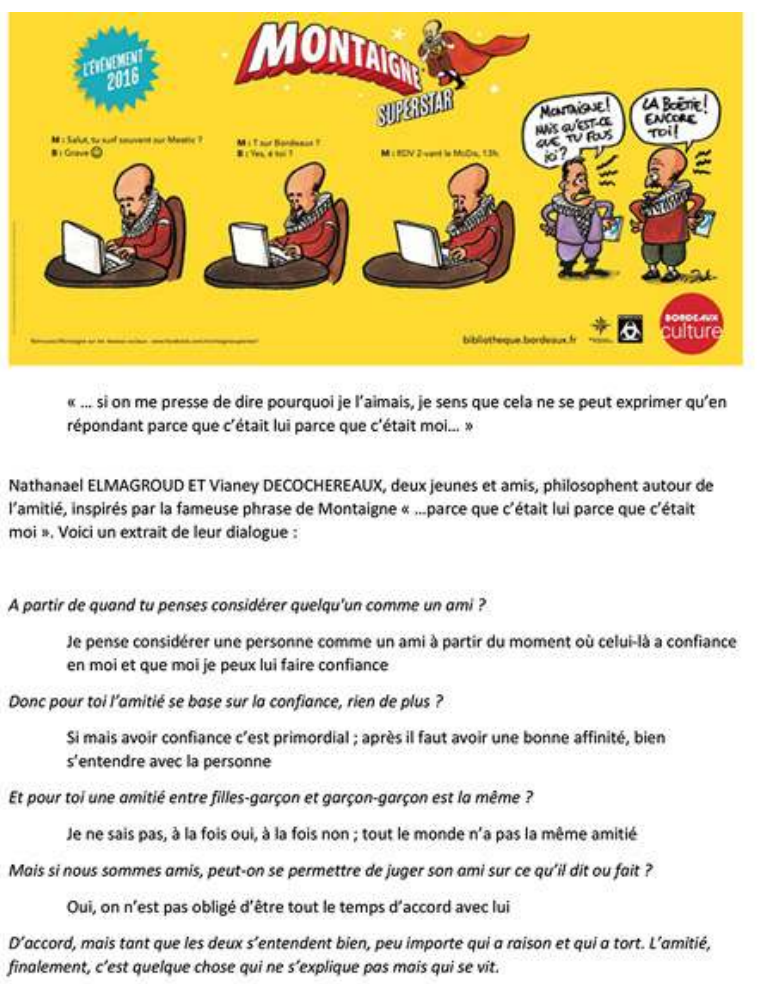

La première constatation est, qu'à l'inverse de l'univers décalé proposé et créé à partir de l'image d'un Montaigne en super héros réalisée par le dessinateur de bande dessinée Jul, les ateliers pédagogiques adoptent une forme traditionnelle et s'appuient sur un discours descendant où le ton scolaire prime. Il s'agit avant tout pour les bibliothécaires de suivre les séquences de l'exposition et de donner aux élèves un maximum d'informations sur la vie de Montaigne. N'exploitant pas les dispositifs numériques proposés à la consultation au sein du parcours, les bibliothécaires interagissent finalement assez peu avec les lycéens qui sont placés dans une posture relativement passive, si l'on excepte le fait de devoir remplir le quizz... Ce contraste est d'ailleurs fortement relevé par les lycéens qui ont apprécié la ligne éditoriale de l'exposition, avec l'illustration de Jul et la scénographie du parcours où la dimension humaine de Montaigne était particulièrement mise en avant, mais ont jugé la visite "ennuyeuse" car trop peu "interactive"7. Le terme d'atelier pédagogique, utilisé par les enseignants comme par les bibliothécaires, amène de plus une certaine confusion puisque les élèves s'attendaient à "faire quelque chose de concret", ce qui prouve en définitive leur envie d'être actifs. D'autre part, malgré de nombreux contenus consultables en ligne, aucun des enseignants interrogés n'avait préparé en amont la visite de l'exposition. La posture attentiste de leurs professeurs a renforcé, chez les élèves, un certain trouble et beaucoup auraient préféré préparer la visite, en commençant par exemple à travailler sur le quizz en classe, afin d'éveiller leur curiosité et d'avoir ainsi moins d'informations à assimiler en un temps très court.

21 Ces quelques observations rapidement esquissées permettent de poser de premiers éléments de réflexion quant à l'usage d'une stratégie transmédiatique pour valoriser un patrimoine dans le cadre d'une activité pédagogique. Il est par exemple intéressant de constater que l'exposition a été pensée, par les enseignants, comme le point d'entrée unique dans l'univers élaboré par la bibliothèque, occultant par là même l'ensemble des autres contenus développés sur le web. Cette absence de prise en compte de l'aspect transmédia du projet, si elle peut s'expliquer par son aspect expérimental et la montée en 
compétence progressive des bibliothécaires dans l'élaboration de telles pratiques de médiation (Bideran et Bourdaa, 2017), s'est ressentie dans la démarche pédagogique par l'absence de collaboration entre bibliothécaires, élèves et enseignants tout comme par un usage relativement dissocié et sporadique des différents contenus produits. L'intérêt des publics scolaires pour le patrimoine écrit étant limité et leur niveau d'attention ayant tendance à diminuer lors d'une visite pédagogique, l'activation des principes du transmédia (co-création et co-participation) aurait pourtant pu permettre de contourner ces difficultés en engageant ces derniers dans la narration.

Créer de l'appétence pour le patrimoine écrit en humanisant ces illustres auteurs est un premier pas intéressant franchi par la bibliothèque. Mais la manière d'engager les publics dans la quête d'informations patrimoniales, historiques ou littéraires reste semble-t-il à façonner. Or les actions pédagogiques, parce qu'elles proposent des publics cibles et s'appuient sur des enseignants, offrent un cadre pertinent pour la co-création de contenus. La mise en place d'une telle stratégie suppose toutefois un déplacement des missions de chacun, et notamment des experts qui ne dispensent plus un discours érudit, mais développent plutôt une attitude constructiviste explicitant le contexte et le dispositif global afin que les élèves recherchent et construisent par eux-mêmes les éléments complémentaires à implémenter à l'univers narratif élaboré...

\section{Conclusion}

Si la discussion avec les publics et leur participation commencent et se poursuivent désormais aussi sur les réseaux numériques selon une temporalité complexe qui dépasse celle du moment de l'exposition et de la visite, les principes d'engagement proposés par une narration transmédia semblent permettre d'aller plus loin dans l'appropriation de contenus.

Pour autant, ces principes sont encore loin d'être complètement intégrés par les professionnels du patrimoine pour qui la construction d'un univers narratif multisupports reste complexe à mettre en œuvre. De conservateurs du patrimoine écrit, les bibliothécaires se sont ici mués en narrateurs ; il leur reste maintenant à se transformer en chef d'orchestre afin de coordonner des médias variés - étant entendu qu'une exposition est aussi un média - d'organiser des collaborations avec différents publics, d'inciter aux échanges entre les communautés concernées, d'imbriquer les multiples contenus produits et d'assurer la cohérence de l'ensemble...

\section{BIBLIOGRAPHIE}

Bideran, J. (de) et Bourdaa, M. Quand les experts du patrimoine s'emparent du transmédia storytelling, Communication et organisation, $\mathrm{n}^{\circ} 51,2017 \mathrm{http}: / /$

communicationorganisation.revues.org/5510 
Favard, F. Comment allier patrimoine et transmedia storytelling ? MediaNum, 2016 https:// medianum.hypotheses.org/86

Jenkins, H., Ito, M. et Boyd, D. Participatory culture in a networked era, Polity, 2016.

Jenkins, H. Convergence Culture. Where old and new media collide. NUY Press, 2006.

Long, G. Transmedia Storytelling, Business, aesthetics and Production at the Jim Henson Company, thèse de doctorat, Massachusetts Insitute of Technology, 2017.

Magro, S. De l'usage des réseaux socio-numériques comme supports d'une médiation culturelle en ligne, La Lettre de l'Ocim, n¹62, 2015, http://ocim.revues.org/1593

Ryan, M.-L. Le transmédia storytelling comme pratique narrative, (traduction Florent Favard), Revue Française des Sciences de l'Information et de la Communication, n¹0, 2017, http://

rfsic.revues.org/2548

\section{NOTES}

1. Pour plus de détails sur cet état de l'art, voir : Favard, F. Comment allier patrimoine et transmedia storytelling? MediaNum, 2016, https://medianum.hypotheses.org/86.

2. Développé entre 2015 et 2016 à l'occasion du tricentenaire de la mort de Louis XIV, il s'agit d'un dispositif transmédia qui se déploie autour d'une exposition temporelle, d'un blog, de fil twitter et d'événements in situ de type concerts : www.leroiestmort.com.

3. Développé en 2015 , ce dispositif transmédia se déploie principalement autour d'un docufiction produit par France TV et d'une bande dessinée interactive : www.lederniergaulois.fr

4. Pour en savoir plus, http://cathedrale.arte.tv/

5. Pour en savoir plus, http://lachasseauxlegendes.com/

6. Cette étude a été réalisée dans le cadre d'un projet pédagogique avec des étudiantes de Master Communication et Générations de l'université Bordeaux Montaigne. Ont été combinés des outils d'évaluation sociologique (questionnaires et focus groupes) avec des outils d'évaluation ethnologique (observation dynamique des séances de visite selon une série d'items). Ont été interrogés : 1 ) des élèves de classes de $1^{\text {re }} \mathrm{L}: 2$ classes divisées en 4 groupes, soit une cinquantaine de lycéens de première L, âgés de 16 à 18 ans ;2) des enseignants accompagnateurs, soit 2 professeures de Français, 1 professeur d'Histoire et 1 professeure documentaliste ; 3) les médiatrices de l'exposition responsables des ateliers pédagogiques, soit 3 bibliothécaires.

7. Les termes entre guillemets sont extraits des propos que nous avons pu recueillir lors des entretiens et des focus group.

\section{RÉSUMÉS}

À partir d'une exposition organisée par la bibliothèque municipale de Bordeaux, les auteures analysent l'adaptation, dans l'univers du patrimoine et des musées, du concept de narration transmédia - envisagé comme nouvelle stratégie de valorisation et nouvelle pratique de communication - pointant les contraintes spécifiques de ce milieu et les difficultés rencontrées pour engager les publics dans une co-construction et une co-participation. 
INDEX

Mots-clés : Exposition, médiation, bibliothèque

\section{AUTEURS}

\section{JESSICA DE BIDERAN}

ingénieure de recherche à l'université Bordeaux Montaigne

jessica.debideran@gmail.com

\section{MÉLANIE BOURDAA}

maître de conférences en sciences de l'information et de la communication à l'université Bordeaux Montaigne

melaniebourdaa@yahoo.fr

\section{JUSTINE DUJARDIN}

conservateur des bibliothèques, responsable des Fonds patrimoniaux de la bibliothèque municipale de Bordeaux

just.dujardin@gmail.com 